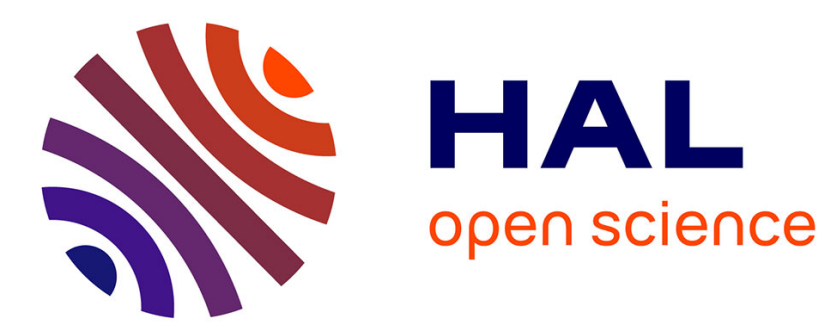

\title{
Non-ideality in multicomponent diffusion of polyelectrolyte solutions
}

L. Belloni, M. Drifford, P. Turq

\section{To cite this version:}

L. Belloni, M. Drifford, P. Turq. Non-ideality in multicomponent diffusion of polyelectrolyte solutions. Journal de Physique Lettres, 1985, 46 (5), pp.207-215. 10.1051/jphyslet:01985004605020700 jpa00232501

\section{HAL Id: jpa-00232501 https://hal.science/jpa-00232501}

Submitted on 1 Jan 1985

HAL is a multi-disciplinary open access archive for the deposit and dissemination of scientific research documents, whether they are published or not. The documents may come from teaching and research institutions in France or abroad, or from public or private research centers.
L'archive ouverte pluridisciplinaire HAL, est destinée au dépôt et à la diffusion de documents scientifiques de niveau recherche, publiés ou non, émanant des établissements d'enseignement et de recherche français ou étrangers, des laboratoires publics ou privés. 
Classification

Physics Abstracts

$36.20-66.10-82.70$

\title{
Non-ideality in multicomponent diffusion of polyelectrolyte solutions
}

\author{
L. Belloni, M. Drifford \\ Département de Physico-Chimie, CEN Saclay, 91191 Gif sur Yvette Cedex, France
}

and P. Turq

Laboratoire d'Electrochimie, Université Pierre et Marie Curie, Paris V, 75230 Paris Cedex, France

(Reçu le 28 novembre 1984, accepté le 9 janvier 1985)

\begin{abstract}
Résumé. - La théorie des modes normaux incluant les écarts à l'idéalité et les interactions hydrodynamiques est développée pour étudier la dynamique des solutions de polyélectrolytes. L'influence de la diffusion non instantanée des petits ions est mise en évidence. Dans le cas des faibles interactions Coulombiennes entre ions ponctuels, les lois classiques des électrolytes simples sont retrouvées. Un autre cas limite redonne le traitement en développement du viriel.
\end{abstract}

\begin{abstract}
The normal-mode theory including corrections for non-ideality and hydrodynamic interactions has been developed for the study of dynamics in polyelectrolyte solutions. The influence of the non-instantaneous diffusion of the small ions is shown. For the case of weak Coulombic interactions between point ions, the classical laws for simple electrolytes are obtained. Another limiting case gives the virial expansion treatment.
\end{abstract}

\section{Introduction.}

The dynamic behaviour of polyelectrolyte solutions, such as some biological solutions, micellar solutions or colloidal suspensions, has been studied in recent years with two apparently different models.

The first one, which can be called the " polymer » theory, consists in calculating, as for polymer solutions, virial coefficients. The hard-core repulsion is complemented with a screened Coulombic repulsion and, eventually, with a Van-der-Waals attraction [1]. This point of view implies an excess of supporting electrolyte and supposes that the diffusion of the small ions is instantaneous. This is a single particle model, the small ions are only involved in the Debye screening parameter.

The second theory comes from electrochemistry and consists in extending the formalism of simple electrolytes, such as salt solutions, to the case of solutions of multicharged macroions. It takes the coupling between the different diffusion modes into account and introduces wellknown concepts like Debye relaxation and Nernst-Hartley diffusion [2-7]. Therefore, this formalism was formerly used in the framework of the Debye-Hückel model which implies weak Coulombic interactions between point-like ions. These approximations are acceptable for simple electrolytes but fail for polyelectrolytes. 
In the present work we have studied the dynamic behaviour of polyelectrolyte solutions by normal-mode analysis with non-ideality corrections and hydrodynamic interactions. We first present the general formalism which considers the time evolution of concentration fluctuations or of dynamic partial structure factors. Then, we calculate the first cumulant of the autocorrelation function for the intensity of scattered light. We show that the effective diffusion coefficient depends on the experimental time resolution. In approximate cases, we recover the two limiting theories.

\section{Theory.}

The equations which govern the time evolution of the density of each species $i$ present in the solution $(i=1, n)$ can be introduced in two different ways.

The first, using the phenomenological approach of Berne and Pecora [2], consists in writing the macroscopic hydrodynamic equations for each particle-fluid (thermodynamic limit) [2-7] taking the hydrodynamic and non-ideality corrections into account.

Instead of using this macroscopic description, we prefer to use, here, the following approach which is more direct and elegant. Moreover, it elucidates the different approximations which one has to introduce.

From the well-known Smoluchowski equation (microscopic description), the standard Mori projection operator technique yields equations for the average density [8-10]. We just give the last result of this treatment. For more details, the reader is referred to published articles. We use the matrix notation of references [9] and [10]. In the absence of chemical reactions,

$$
\frac{\partial \mathbf{C}}{\partial t}(q, t)=-\mathbf{\Omega} 1(q) \mathbf{C}(q, t)
$$

C represents the column matrix $\left(\begin{array}{c}C_{1} \\ C_{2} \\ \vdots \\ \vdots\end{array}\right)$ where $C_{i}(q, t)$ is the Fourier transform of the concentration fluctuation of species $i$. The frequency matrix $\Omega 1(q)$ is [10]

$$
\mathbf{\Omega} 1(q)=q^{2} \mathbf{H} 1(q) \boldsymbol{\sigma}^{-1}(q)
$$

where the interaction matrix $\boldsymbol{\sigma}(q)$ is related to the static structure factor matrix $\mathbf{S}(q)$ by

$$
\sigma_{i j}(q)=\left(c_{i} c_{j}\right)^{1 / 2} S_{i j}(q)
$$

where $c_{i}$ represents the mean concentration of species $i$. The factor $\left(c_{i} c_{j}\right)^{1 / 2}$ comes from our definition of a partial structure factor :

$$
S_{i j}(q)=\delta_{i j}+\left(c_{i} c_{j}\right)^{1 / 2} \int\left(g_{i j}(r)-1\right) \mathrm{e}^{i \mathbf{q} \cdot \mathbf{r}} \mathrm{d} \mathbf{r}
$$

where $g_{i j}$ is the partial pair distribution function.

The hydrodynamic matrix $\mathbf{H} 1(q)$ is not in general a simple expression of the distribution functions. Indeed, the drag that particle $i$ induces on particle $j$ depends on the relative position of $i$ and $j$ but also on the positions of the other particles $k$. Even if this last dependence is ignored, the hydrodynamic term is not known exactly. Many publications exist on this important problem $[11,12]$. We just give, here, the limiting expression, valid for long interparticle distances :

$$
H 1_{i j}(q)=c_{i}\left(D_{i} \delta_{i j}+k T c_{j} \int\left(g_{i j}-1\right) T_{z z} \mathrm{e}^{i \mathbf{q} \cdot \mathbf{r}} \mathrm{dr}\right) .
$$


$D_{i}$ is the diffusion coefficient of the free particle $i, T_{z z}$ represents the component along $\mathbf{q}$ of the Oseen tensor $\mathbf{T}(r)=(8 \pi \eta r)^{-1}\left(1+\mathbf{r} \times \mathbf{r} / r^{2}\right)$. We note that with expression (5) the self-diffusion coefficient of the polyion is constant. It is possible to use a more elaborate coupling tensor instead of the Oseen tensor [11, 12].

Equation (1) supposes two important approximations :

i) small deviations from the equilibrium state are considered, which corresponds to the linearization in the Berne and Pecora treatment;

ii) the effect of the memory function is neglected, which means that the so-called relaxation effect is not present in this theory. Expression (1) is then correct in the short time limit $(t \rightarrow 0)$. In this case, the concentration fluctuations decay as a sum of exponentials. The study of the long-time behaviour requires an approximate expression for the memory function [8].

It is important to note that equation (1) with definitions (2) to (5) reduces to the thermodynamic model in the limit $q \rightarrow 0$. Indeed, the term $i j$ of the matrix $\sigma^{-1}(q=0)$ is nothing else than $\left.\frac{\partial \beta \mu_{i}}{\partial c_{j}}\right|_{c_{k}}$ where $\beta=(k T)^{-1}$ and $\mu_{i}$ is the chemical potential of species $i$ [13]. For the case of an electrolyte solution, the electrical force comes from the particular form of the matrix $\mathbf{S}(q)$ : the electroneutrality condition assures that the determinant of $\mathbf{S}(0)$ is zero. Therefore, the matrix $\boldsymbol{\sigma}^{-1}(q \rightarrow 0)$ seems to be undefined. However, this is only an apparent paradox, as is seen by using the more adequate matrix $\hat{\mathbf{C}}(q)$ where $\hat{C}_{i j}(q)$ is the Fourier transform of the direct correlation function $C_{i j}(r)$

$$
\hat{C}_{i j}(q)=\left(c_{i} c_{j}\right)^{1 / 2} \int C_{i j}(r) \mathrm{e}^{i \mathbf{q} . \mathbf{r}} \mathrm{dr} .
$$

We recall that $\mathbf{S}(q)$ is related to $\hat{\mathbf{C}}(q)$ by the Ornstein-Zernike equation [14]

$$
\mathbf{S}(q)(\mathbf{I d}-\mathbf{C}(q))=\mathbf{I d}
$$

and then

$$
\sigma_{i j}^{-1}(q)=\left(\delta_{i j}-\hat{C}_{i j}(q)\right) /\left(c_{i} c_{j}\right)^{1 / 2}
$$

Due to the long distance behaviour of the Coulombic potential, the functions $\hat{C}_{i j}(q)$ exhibit a well-known divergence for $q \rightarrow 0$,

$$
-q_{i j} / q^{2} \text { where } q_{i j}=4 \pi L_{\mathrm{B}}\left(c_{i} c_{j}\right)^{1 / 2} Z_{i} Z_{j}
$$

$\left(L_{\mathrm{B}}=e^{2} /\left(4 \pi \varepsilon_{0} \varepsilon k T\right)\right.$ is the Bjerrum length, $Z_{i}$ is the charge of $i, \sum_{i} Z_{i} c_{i}=0$ is the electroneutrality condition). The expansion of $\hat{C}_{i j}(q)$ for small $q$ is :

$$
\hat{C}_{i j}(q) \sim-q_{i j} / q^{2}+C_{i j}^{\prime}+C_{i j}^{\prime \prime} q^{2}+\cdots .
$$

We then recognize in equation (8) three terms :

$-\delta_{i j}$ represents the ideal term of free diffusion.

$-q_{i j} / q^{2}$ is the electric term.

$-C_{i j}^{\prime}$ is the non-ideality term. This term disappears in the Debye-Hückel approximation.

The electric part of the frequency matrix (2) is then of zero order in $q^{2}$ when the non-ideality expression is linear in $q^{2}$. We recall that this distinction (necessary for $q \rightarrow 0$ ) is clear with $\hat{\mathbf{C}}(q)$ but not with $\mathbf{S}(q)$. If one wants to preserve this separation for all values of $q$, it is important to take the Debye-Hückel term out of the non-ideality term.

In the absence of hydrodynamic interaction $\left(H 1_{i j}=c_{i} D_{i} \delta_{i j}\right)$ and non-ideality correction 
$\left(C^{\prime}=0\right)$, we find the well-known equations which are classically established with the ideal chemical potential and the electric field [2-7] :

$$
\frac{\partial C_{i}}{\partial t}(q, t)=-D_{i} \sum_{k}\left(q^{2} \delta_{i k}+\left(\frac{c_{i}}{c_{k}}\right)^{1 / 2} q_{i k}\right) C_{k}(q, t) .
$$

The equations (1) are solved with Laplace transformation. One calculates the eigenvalues of the matrix $\mathbf{\Omega 1}(q)$ which gives the different normal modes. Each concentration fluctuation $C_{i}(q, t)$ decays as a sum of exponentials.

The interesting results depend on the experimental method. Some measurements are focused on the slower mode, and others on the initial slope $\frac{\partial C_{i}}{\partial t}(q, 0)$. In the following formalism we consider the autocorrelation function of the intensity scattered by different forms of radiation such as light, X-ray or neutron.

\section{Autocorrelation function of the scattered intensity.}

The function of interest is now the autocorrelation function $C_{i}(q, t) C_{j}(q, 0) \sim\left(c_{i} c_{j}\right)^{1 / 2} S_{i j}(q, t)$, where $S_{i j}(q, t)$ is the dynamic partial structure factor. We deduce easily :

$$
\frac{\partial \mathbf{S}}{\partial t}(q, t)=-\mathbf{\Omega}(q) \mathbf{S}(q, t)
$$

where

$$
\mathbf{\Omega}(q)=q^{2} \mathbf{H}(q) \mathbf{S}^{-1}(q) \quad \text { and } \quad H_{i j}(q)=D_{i} \delta_{i j}+k T\left(c_{i} c_{j}\right)^{1 / 2} \int\left(g_{i j}-1\right) T_{z z} \mathrm{e}^{i \mathbf{q} \cdot \mathbf{r}} \mathrm{d} \mathbf{r} .
$$

It can be shown that this equation only supposes the suppression of the memory effect and not the linearization [8-10].

Equation (11) can be solved in a way similar to equation (1). The eigenvalues of $\boldsymbol{\Omega} 1$ and $\boldsymbol{\Omega}$ are identical. The result is of the form [9]

$$
\mathbf{S}(q, t)=\mathbf{M}(q, t) \mathbf{S}(q)
$$

where $\mathbf{M}(t)=\sum_{j} \mathbf{m}_{j} \mathrm{e}^{-\Gamma_{j} t} . \Gamma_{j}$ is the $j$ th eigenvalue of $\Omega, \mathbf{m}_{j}$ its $j$ th eigenmatrix.

The expression of the $\Gamma_{j}$ and $\mathbf{m}_{j}$ as a function of the coefficients of the matrix $\boldsymbol{\Omega}$ are easily found but are very long and heavy, especially in the presence of many ionic species, hydrodynamic interactions and non-ideality terms ! However, the behaviour of $\mathbf{S}(q, t)$ for small values of $t$ is clearer.

3.1 InItIAL SLOPE $\left(t \ll \Gamma_{j}^{-1}, j=1, n\right)$. - If we study the true slope of $\mathbf{S}(q, t)$ at the origin, we find :

$$
\frac{\partial \mathbf{S}}{\partial t}(q, 0)=-\mathbf{\Omega S}(q, 0)=-q^{2} \mathbf{H}(q) \mathbf{S}^{-1}(q) \mathbf{S}(q)=-q^{2} \mathbf{H}(q)
$$

For the common case of a polyelectrolyte plus an added salt solution containing polyion (1), counterion (2) and coion (3),

$$
\begin{aligned}
\frac{\partial S_{11}}{\partial t}(q, 0) & =-q^{2}\left(D_{1}+k T c_{1} \int\left(g_{11}-1\right) T_{z z} \mathrm{e}^{i \mathbf{q} \cdot \mathbf{r}} \mathrm{d} \mathbf{r}\right) \\
& =-q^{2} D_{1}(1+H(q))
\end{aligned}
$$


If the scattered intensity $I(q, t)$ results principally from the macroions, the much used first cumulant $K=-\frac{\partial \log I(q, 0)}{\partial t}$ is equal to

$$
K=q^{2} D_{1}(1+H(q)) / S_{11}(q) .
$$

Since the memory function has no effect at $t=0$, this result is exact. It has been found by several authors $[15,16]$.

If we take the contribution of small ions to the intensity into account, we must introduce the partial form factor $F_{i j}(q)$ and

$$
K=q^{2} \frac{\sum_{i, j}\left(c_{i} c_{j}\right)^{1 / 2} F_{i j}(q) H_{i j}(q)}{\sum_{i, j}\left(c_{i} c_{j}\right)^{1 / 2} F_{i j}(q) S_{i j}(q)} .
$$

It is important to note that in this case the first cumulant depends linearly on $q^{2}$ for small values of $q$. It is not surprising that it does not give the Debye relaxation mode since for $t \rightarrow 0$ the electroneutrality condition has no effect.

This initial slope can be studied by the neutron spin-echo experiment [17]. The characteristic time $\tau$ is much smaller than the inverse of all the eigenvalues of $\boldsymbol{\Omega}$.

In dynamic light scattering, $\tau$ is too long to measure the initial slope exactly and expressions (15) or (16) no longer correspond to the experimental first cumulant.

3.2 INTERMEDIATE SLOPE $\left(t \gg \Gamma_{1}^{-1}\right.$ AND $\left.t \ll \Gamma_{2}^{-1} \ldots\right)$. - For the case of light scattering. where $q \rightarrow 0$, namely $q^{2} \ll q_{i i}$, it is known that there are one relaxation mode or Debye mode $\Gamma_{1}\left(\Gamma_{1}=\sum_{i} D_{i} q_{i i}\right.$ in absence of hydrodynamic term $)$ independent of $q$, which corresponds to the electroneutrality effect, and $n-1$ diffusion modes $\left(D q^{2}\right)$. The relaxation mode is too fast and cannot be detected by the correlator. Therefore, it is necessary to calculate the slope for $t=\tau$, with $\tau \gg \Gamma_{1}^{-1}$ and $\tau \ll \Gamma_{2}^{-1}, \Gamma_{3}^{-1}, \ldots$, namely

$$
\frac{\partial \mathbf{S}}{\partial t}(q, \tau)=-\sum_{j=2}^{n} \mathbf{m}_{j} \Gamma_{j} \mathbf{S}(q) .
$$

The sum does not take the first exponential into account.

The intermediate slope can be obtained without calculating the different eigenvalues $\Gamma_{j}$ and eigenmatrix $\mathbf{m}_{j}$ : (we use the notation of Ref. [9]).

and

$$
\mathbf{\Omega}=\mathbf{Q} \operatorname{Diag}\left[\Gamma_{1}, \Gamma_{2}, \ldots\right] \mathbf{Q}^{-1}
$$

$$
\mathbf{m}_{j}=\mathbf{Q} \mathbf{d}_{j} \mathbf{Q}^{-1}
$$

where $\mathbf{d}_{j}$ is the matrix with all elements equal to zero except the $j$ th diagonal element, which is equal to 1 .

A simple matrix manipulation of equations (18) and (19) gives

Now,

$$
\Gamma_{j} \mathbf{m}_{j} \mathbf{S}(q)=q^{2} \mathbf{m}_{j} \mathbf{H}(q) .
$$

$$
\frac{\partial S}{\partial t}(q, \tau)=-q^{2} \sum_{j=2}^{n} \mathbf{m}_{j} \mathbf{H}(q)=-q^{2}\left(\mathbf{I d}-\mathbf{m}_{1}\right) \mathbf{H}(q) .
$$


The linear development of the cumulant in $q^{2}$ depends on the value of $\mathbf{m}_{1}(q=0)$. At $q=0$, $\operatorname{Diag}\left[\Gamma_{1}, \Gamma_{2}, \ldots\right]$ restricts to $\Gamma_{1}(0) \mathbf{d}_{1}(0)$ and thus $\mathbf{m}_{1}(q=0)=\Omega(0) / \Gamma_{1}(0)$. Then,

$$
\frac{\partial S}{\partial t}(q, \tau) \underset{q \rightarrow 0}{\longrightarrow}-q^{2}\left(\mathbf{I d}-\mathbf{\Omega}(0) / \Gamma_{1}(0)\right) \mathbf{H}(0)
$$

This expression is very simple since $\boldsymbol{\Omega}(0)=\lim _{q \rightarrow 0} q^{2} \mathbf{H}(q) \mathbf{S}^{-1}(q)=\mathbf{H}(0) \mathbf{Q}_{\mathrm{DH}}\left(\mathbf{Q}_{\mathrm{DH}}\right.$ is constituted by the elements $q_{i j}$ ) and $\Gamma_{1}(0)$ is equal to the trace of the matrix $\Omega(0)$.

We note that $\partial S(0, \tau) / \partial t$ does not depend on the non-ideality term $\left(C^{\prime}\right)$ but only depends on the Debye screening parameters $q_{i j}$ and on the hydrodynamic perturbation.

If the light is only scattered by the polyions, then the experimental first cumulant is

$$
K=\lim _{q \rightarrow 0} \frac{\frac{\partial S_{11}}{\partial t}(q, \tau)}{S_{11}(0,0)}
$$

We note that $S_{11}(0, \tau)$ differs from $S_{11}(0,0)$ by a factor in $q^{2}$.

In the absence of hydrodynamic interactions, the effective diffusion coefficient $D\left(=K / q^{2}\right)$ is

$$
D=D_{1} \frac{\sum_{i=2}^{n} D_{i} q_{i i}}{\sum_{i=1}^{n} D_{i} q_{i i}} \mid S_{11}(0)
$$

If, for the hydrodynamic perturbation, one takes only the polyion-polyion term into account, then equation (24) remains true with $D_{1}$ replaced by $D_{1}(1+H(0))$.

Expression (24) gives two important limiting values :

i) Absence of salt :

$$
D=\frac{D_{1} D_{2} q_{22}}{D_{1} q_{11}+D_{2} q_{22}} / S_{11}(0) \quad \text { with } \quad S_{11}(0)=\frac{q_{22}}{q_{11}+q_{22}} \frac{\chi_{\mathrm{T}}}{\chi_{\mathrm{T}}^{0}} .
$$

The second equality of expression (25) is a classical thermodynamic relation [13]. We recognize the compressibility $\chi_{\mathrm{T}}$ and its ideal value $\chi_{\mathrm{T}}^{0}$. Now,

$$
D=\frac{D_{1} D_{2}\left(q_{11}+q_{22}\right)}{D_{1} q_{11}+D_{2} q_{22}} \frac{\chi_{\mathrm{T}}^{0}}{\chi_{\mathrm{T}}}=\frac{D_{1} D_{2}\left(q_{11}+q_{22}\right)}{D_{1} q_{11}+D_{2} q_{22}}\left(1+\frac{\mathrm{d} \log \gamma_{ \pm}}{\mathrm{d} \log c}\right) .
$$

Equation (26) exhibits the known Nernst-Hartley diffusion coefficient [2] with the correction in the activity coefficient.

ii) Excess of salt :

Supposing that the polyelectrolyte concentration is much smaller than the salt concentration $c_{\mathrm{s}}$, then the linear behaviour in $c_{1}$ of expression (24) is given by

$$
D \sim D_{1}\left(1-c_{1} \hat{h}_{11}-\frac{D_{1} q_{11}}{D_{2} q_{22}+D_{3} q_{33}}\right)
$$

where $\hat{h}_{11}=\int\left(g_{11}^{0}-1\right) \mathrm{d} \mathbf{r}$ and $g_{11}^{0}(r)$ represents the infinite dilution limit of $g_{11}(r)$ for $c_{1}=0$. 
If we suppose that $D_{1} \ll D_{2}, D_{3}$, then

$$
D \sim D_{1}\left(1+k_{\mathrm{D}} c_{1}\right)
$$

where

and

$$
k_{\mathrm{D}}=k_{\mathrm{S}}-k_{\mathrm{H}}
$$

$$
k_{\mathrm{S}}=\int\left(1-g_{11}^{0}\right) \mathrm{d} \mathbf{r} ; \quad k_{\mathrm{H}}=\int\left(1-g_{11}^{0}\right) k T T_{z z} / D_{1} \mathrm{~d} \mathbf{r} .
$$

The exact value of $g_{11}^{0}(r)$ is $\exp \left(-\beta v_{11}(r)\right)$ where $v_{11}(r)$ is the pair potential. For the case of charged hard-spheres (diameter $\sigma=2 \AA$ ), it is natural to choose

$$
\begin{aligned}
\beta v_{11}(r) & =+\infty & & r<\sigma \\
& =\frac{Z_{1}^{2} L_{\mathrm{B}}}{(1+\kappa A)^{2}} \frac{\mathrm{e}^{-\kappa(r-\sigma)}}{r} & & r>\sigma
\end{aligned}
$$

with $\kappa^{2}=q_{22}+q_{33}\left(q_{22}\right.$ includes only the counterions of the salt).

We recognize the theoretical treatment which is used by many authors including Corti and Degiorgio $[1,18,19]$. It gives the initial slope of $D$ versus $c_{1}$. We note that the approximation $D_{1} \ll D_{2}, D_{3}$ can easily be suppressed without complicating expression (27).

It should be noticed that, if the non-linear dependence of $D$ versus $c_{1}$ is required, it is not sufficient to use equations (28) to (30) corrected by adding the contribution of the new counterions to the screening parameter $\kappa$.

The Debye-Hückel approximation of equation (27) consists of linearizing $\exp \left(-\beta v_{11}(r)\right)$ and suppressing the hard-sphere core in the expression for the potential (30) :

$$
D \sim D_{1}\left(1+\frac{q_{11}}{\kappa^{2}}-\frac{D_{1} q_{11}}{D_{2} q_{22}+D_{3} q_{33}}\right) \text {. }
$$

In the case of a monovalent salt,

$$
D \sim D_{1}\left(1+\frac{Z_{1}^{2} c_{1}}{2 c_{\mathrm{s}}}\left(1-\frac{2 D_{1}}{D_{2}+D_{3}}\right)\right)
$$

and if $D_{1} \ll D_{2}, D_{3}, D \sim D_{1}\left(1+Z_{1}^{2} c_{1} / 2 c_{\mathrm{s}}\right)$ which is a well established result [5-7].

In the general case (intermediate salinity, complete hydrodynamic perturbation, total scattered intensity with different form factors), the expression for the effective diffusion coefficient (22) remains simple and can be used without difficulty.

3.3 «LONG TIME SLOPE » $\left\{t \gg \Gamma_{1}^{-1}, \Gamma_{2}^{-1}, t \ll \Gamma_{3}^{-1}\right)$. - For polyelectrolyte solution containing added salt, the first diffusional mode $\Gamma_{2}$ is always close to the diffusion coefficient of the small ions $D_{2}, D_{3}, q^{2}$ when the second $\Gamma_{3}$ is close to the diffusion coefficient of the polyion $D_{1} q^{2}$. In some cases, the experimental resolution cannot detect the second exponential which is too fast, particularly if $D_{2}, D_{3} \gg D_{1}$. The experimental first cumulant is then identical to the slow mode $\Gamma_{3}[7,20]$. Recall that $\Gamma_{3}$ is not the exact long time behaviour since the memory effect has been neglected (experimental curve $I(0, t)$ does not seem to be a pure exponential).

In ionic micellar solutions having a low critical micellar concentration, $D_{2}$ and $D_{3}$ are in general the coefficients of small ions $\left(\mathrm{Na}^{+}, \mathrm{Cl}^{-} \ldots\right)$ and $\Gamma_{2}$ is large. When the cmc is large, $\Gamma_{2} / q^{2}$ is close to the diffusion coefficient of the monomer and becomes more measurable.

In all cases, it is important to evaluate the number of normal modes that the experimental 
method can detect. If all diffusional modes are analysed, then expression (22) is valid. In the opposite case, it is necessary to calculate the smaller eigenvalue of $\boldsymbol{\Omega}(q)$. This calculation is not mathematically difficult but becomes rapidly tedious. We just give the result for the case of an excess of salt $\left(c_{1} \rightarrow 0\right)$ and in the absence of hydrodynamic interaction :

$$
D=\Gamma_{3} / q^{2} \sim D_{1}\left(1-c_{1} \hat{h}_{11}-D_{1} \frac{D_{1} \frac{q_{11}}{q_{23}} S_{23}^{0}+D_{2}\left(S_{12}^{0}\right)^{2}+D_{3}\left(S_{13}^{0}\right)^{2}}{D_{2} D_{3}+D_{1} \frac{S_{23}^{0}}{q_{23}}\left(D_{2} q_{22}+D_{3} q_{33}\right)}\right)
$$

$S_{23}^{0}$ is the zero order (in $c_{1}$ ) of $S_{23}, S_{12}^{0}$ and $S_{13}^{0}$ are the first order of $S_{12}$ and $S_{13}$ respectively, namely, $S_{12}^{0}=\left(c_{1} c_{2}\right)^{1 / 2} \int\left(g_{12}\left(c_{1}=0\right)-1\right) \mathrm{dr}$. Expression (33) is identical to (27) if $D_{1} \ll D_{2}$,
$D_{3}$.

With the Debye-Hückel structure factors, the expression becomes (monovalent salt)

$$
D \sim D_{1}\left(1+\frac{Z_{1}^{2} c_{1}}{2 c_{\mathrm{s}}}\left(1-D_{1} \frac{\frac{D_{2}+D_{3}}{2}-D_{1}}{D_{2} D_{3}-D_{1} \frac{D_{2}+D_{3}}{2}}\right)\right)
$$

This expression has been given by several authors [6, 20]. We note that equations (32) and (34) are identical if $D_{2}=D_{3}$. In this case, the polyion-polyion fluctuations have no second mode $\Gamma_{2}$ component [7].

\section{Conclusion.}

In this paper, we have presented the classical normal-mode formalism introducing non-ideality corrections and hydrodynamic perturbations, for the study of dynamic behaviour of polyelectrolyte solutions. It is important to notice that these correction terms are absolutely necessary for such solutions having strong interactions. We have shown that the linear development of the effective diffusion coefficient versus the volume fraction of polyelectrolyte is a limiting case of this general theory. We have also pointed out that the notion of an experimental first cumulant is difficult to define and depends on the time resolution of the experiment. For the case of intermediate times, we have given a very simple expression of the effective diffusion coefficient.

Several conclusions can be noted :

i) For small values of the vector of diffusion $q$, the first cumulant is always linear in $q^{2}$, even if the true initial slope $\frac{\partial S}{\partial t}(q, 0)$ has been measured. Indeed, in the limit $q=0$, only the Debye relaxation exponential subsists but its practical contribution is zero, owing to the electroneutrality condition.

ii) The different expressions for the effective diffusion coefficient, (15), (24), (26), (27) and (33), are similar only if $D_{1} q_{11} \ll D_{2} q_{22}, D_{3} q_{33}$ (this condition is stronger than $D_{1} \ll D_{2}, D_{3}$ ). For very large polyions, the diffusion coefficient $D_{1}$ is small but the factor $D_{1} Z_{1}$ remains of the same order as $D_{2}$ and $D_{3}$. Then, it is important to take account of the non instantaneous diffusion of the small ions.

iii) There has been a certain controversy between authors using a virial expansion (Eqs. (28) and (29)) $[1,21]$ and those using a Debye-Hückel expansion (Eq. (34)) $[4,7,20]$. By fitting the 
experimental curves of $D$, they do not find the same polyion charge. Since it is analytic and contains no assumption on the shape and size of the macroion, equation (34) seems to be more attractive. On the other hand, equation (28) assumes a spherically symmetric pair potential and an expression for the hydrodynamic tensor. In fact, the expansion in $Z_{1}^{2} c_{1} / 2 c_{\mathrm{s}}$ is based upon the approximations of the Debye-Hückel model, namely the zero size of polyion and the linearization of $\exp \left(-\beta v_{11}(r)\right)$ [1]. The first approximation suppresses hydrodynamic interactions which are really important. Moreover, the linearization is not valid for polyelectrolyte potentials. These effects explain the fact that the charge fitted from the experimental curves with linearized expression (34) is always smaller than those deduced using (28) and (29). It is interesting to note that it is possible to use equation (29) with a zero radius. In this case, the charge deduced is much too large. For some sizes, it happens that the same charge gives similar values of the slope $k_{\mathrm{D}}$ with the two different expressions. It is a reason for which the simple expression (34) is so much used.

The interest of the general theory developed here is that it takes all interactions into account. One of its limitations is that it is necessary to calculate the static distribution functions $g_{i j}(r)$. For spherical particles, one of the most practical and realist models is based on the HNC integral equation $[22,23]$. For non-spherical polyions, good approximations of the $g_{i j}(r)$ do not exist at the moment ; in any case, it seems preferable to approximate the polyion by a sphere rather than to use expression (34). Another problem is the expression of the hydrodynamic interactions for non-dilute solutions [11. 12].

Finally, we recall that the memory effect (or the relaxation effect) is neglected. Several works on the long time behaviour of the dynamics of colloidal solutions seem to be promising [24].

\section{References}

[1] Corti, M. and Degiorgio, V., J. Phys. Chem. 85 (1981) 711.

[2] Berne, B. and Pecora, R., Dynamic Light Scattering (Wiley) 1976.

[3] Stephen, M. J., J. Chem. Phys. 55 (1971) 3878.

[4] Rohde, A. and SaCKmann, E., J. Colloid Interface Sci. 70 (1979) 494.

[5] LiN, S. C., LeE, W. I. and SchurR, J. M., Biopolymers 17 (1978) 1041.

[6] Tivant, P., Turq, P., Drifford, M., Magdelenat, H. and Menez, R., Biopolymers 22 (1983) 643.

[7] Evans, D. F., Mukherjee, S., Mitchell, D. J. and Ninham, B. W., J. Colloid Interface Sci. 93 (1983) 184.

[8] Ackerson, B. J., J. Chem. Phys. 69 (1978) 684.

[9] Akcasu, A. Z., Hammouda, B., Lodge, T. P. and Han, C. C., Macromolecules 17 (1984) 759.

[10] Akcasu, A. Z., Benmouna, M. and Hammouda, B., J. Chem. Phys. 80 (1984) 2762.

[11] Batchelor, G. K., J. Fluid Mech. 74 (1976) 1.

[12] Felderhof, B. U., J. Phys. A 11 (1978) 929.

[13] Rice, S. A. and Nagasawa, M., Polyelectrolytes solutions (Academic Press) 1961.

[14] Hansen, J. P. and McDonald, I. A., Theory of simple liquids (Academic Press) 1976.

[15] Pusey, P. N., J. Phys. A 8 (1975) 664 and 1433.

[16] Ackerson, B. J., J. Chem. Phys. 64 (1976) 242.

[17] HAYTER, J. B., "Theory of Neutron Spin-Echo Spectrometry " in Proc. International Workshop on Neutron Spin Echo, ed. F. Mezei (Springer, Berlin) 1981.

[18] Dorshow, R., Briggs, J., Bunton, C. A. and Nicoli, D. F., J. Phys. Chem. 86 (1982) 2356.

[19] Chattopadhyay, A. K., Belloni, L., Drifford, M. and Dubois, M., to be published.

[20] Mitchell, D. J., Ninham, B. W. and Evans, D. F., J. Colloid Interface Sci. 101 (1984) 292

[21] Degiongio, V. and Corti, M., J. Colloid Interface Sci. 101 (1984) 289.

[22] Elkoubi, D., Turq, P. and Hansen, J. P., Chem. Phys. Lett. 52 (1977) 493.

[23] Rogers, F. J., J. Chem. Phys. 73 (1980) 6272.

[24] Hess, W. and Klein, R., J. Phys. A 15 (1982) L669. 